\title{
CLINICAL-PATHOLOGIC CONFERENCE
}

\section{CLINICAL-PATHOLOGIC CONFERENCE IN THORACIC SURGERY: PROXIMAL PULMONARY ARTERY OBSTRUCTION SECONDARY TO TAKAYASU ARTERITIS}

\author{
Thoralf M. Sundt, MD \\ This case, with added links and enhanced graphics, is available for further study on the World Wide Web at: \\ http://www.ctsnet.org/doc/3196.
}

\author{
Participants \\ From the Washington University School of Medicine \\ Barnes-Jewish Hospital \\ St Louis, Missouri \\ THORACIC SURGERY \\ Dr Joel Cooper \\ Dr Taine Pechet \\ Dr G. Alexander Patterson \\ Dr Charles Roper \\ CARDIAC SURGERY \\ Dr Thoralf Sundt
}

\section{Case presentation}

Dr Pechet: The patient is a 76-year-old woman who was previously in good health except for a history of hyperthyroidism, arthritis, and hypertension. She is a lifelong nonsmoker. She presented with a history of 6 months of progressive weakness, peripheral edema, and dyspnea on exertion to the point that she could no longer walk more than 10 to 15 feet. She had only minimal improvement with a trial of diuretics. Pulmonary function tests demonstrated a forced vital capacity of $74 \%$ and a forced expiratory volume in 1 second of $82 \%$ predicted with an arterial $\mathrm{Po}_{2}$ of $62 \mathrm{~mm} \mathrm{Hg}$ while breathing room air. An electrocardiogram showed right atrial enlargement and right axis deviation. She also had a chest radiograph.

Received for publication July 20, 2000; accepted for publication Aug 4, 2000.

Address for reprints: Thoralf M. Sundt, MD, Department of Cardiothoracic Surgery, Washington University School of Medicine, Queeny Tower, Suite 3106, One Barnes Hospital Plaza, St Louis, MO 63110.

J Thorac Cardiovasc Surg 2001;121:163-75

Copyright (C) 2001 by The American Association for Thoracic Surgery

$0022-5223 / 2001 \$ 35.00+0 \quad \mathbf{1 2 / 1 / 1 1 1 6 5 2}$

doi:10.1067/mtc.2001.111652

\author{
CARDIOTHORACIC ANESTHESIOLOGY \\ Dr Nikolaos Skubas \\ RADIOLOGY \\ Dr Harvey Glazer-Chest Radiology \\ Dr Barry Siegel—Nuclear Medicine \\ Dr Jeffrey Yu-Nuclear Medicine \\ SURGICAL PATHOLOGY \\ Dr Jon Ritter \\ MEDICAL ONCOLOGY \\ Dr Shabbir Safdar
}

Dr Glazer: The chest radiograph shows moderate enlargement of the cardiac silhouette. The aorta is tortuous and mildly dilated. There is right paratracheal widening, which may be due to a dilated superior vena cava (SVC). Enlargement of the main pulmonary artery is seen. The lungs are clear.

Dr Pechet: The patient then had a ventilation-perfusion scan because her physicians were concerned that she had had a pulmonary embolism.

Dr Yu: The ventilation-perfusion images performed in August 1999 demonstrate normal ventilation and globally decreased perfusion to the right lung (Fig 1). There are no segmental perfusion abnormalities to suggest pulmonary embolism. The global decrease suggests a central mass compressing the right pulmonary vasculature and decreasing blood flow. A subsequent ventilation-perfusion study from December 1999 again shows normal ventilation and the same global decrease in perfusion to the right lung (Fig 2). In addition, it demonstrates some peripheral wedge-shaped defects, which raise the possibility of superimposed pulmonary embolism.

Dr Patterson: Dr Pechet, was there some thought that this patient had acute pulmonary emboli?

Dr Pechet: That was the original suspicion that led to the ventilation-perfusion study. Her physicians then obtained a spiral computed tomographic (CT) scan. 


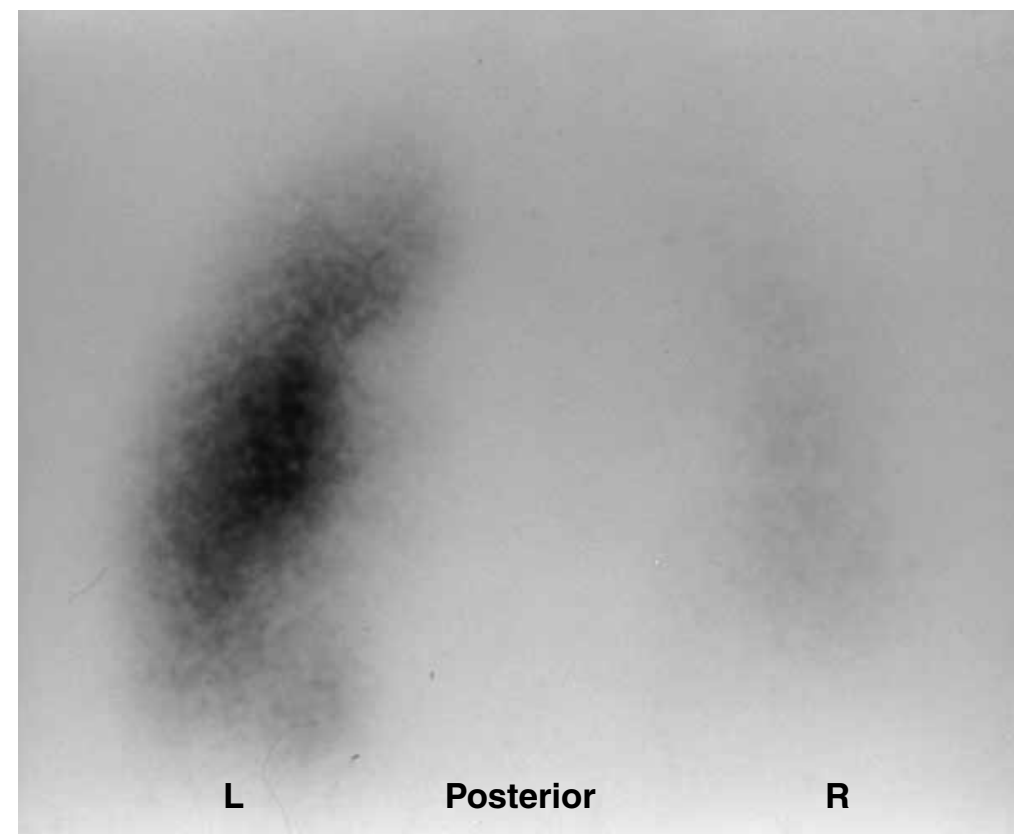

Fig 1. Posterior pulmonary perfusion image in August 1999 demonstrates globally decreased perfusion of the right lung. The ventilation images (not shown) were normal.

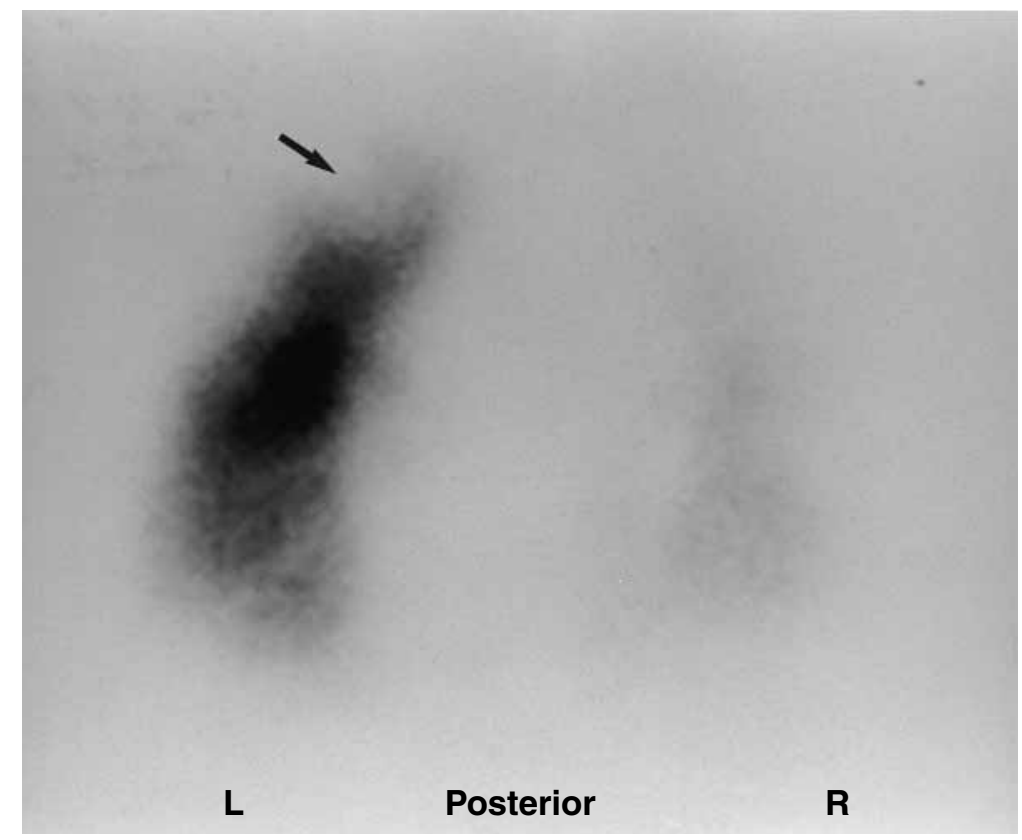

Fig 2. Posterior pulmonary perfusion image in December 1999 shows decreased perfusion of the right lung. A segmental perfusion defect (arrow) is also noted in the apical subsegment of the left upper lobe.

Dr Glazer: The SVC is dilated (Figs 3 and 4). There is focal narrowing and thickening of the wall of the left pulmonary artery. Thickening of the wall of the descending thoracic aorta also is noted. The right pulmonary artery is markedly narrowed with associated wall thickening. Normal sized lymph nodes are seen. No pulmonary emboli are seen. Images of the lung parenchyma are normal. There are no peripheral opacities that might represent peripheral infarcts. The above findings are most consistent with a vasculitis. The dif- 

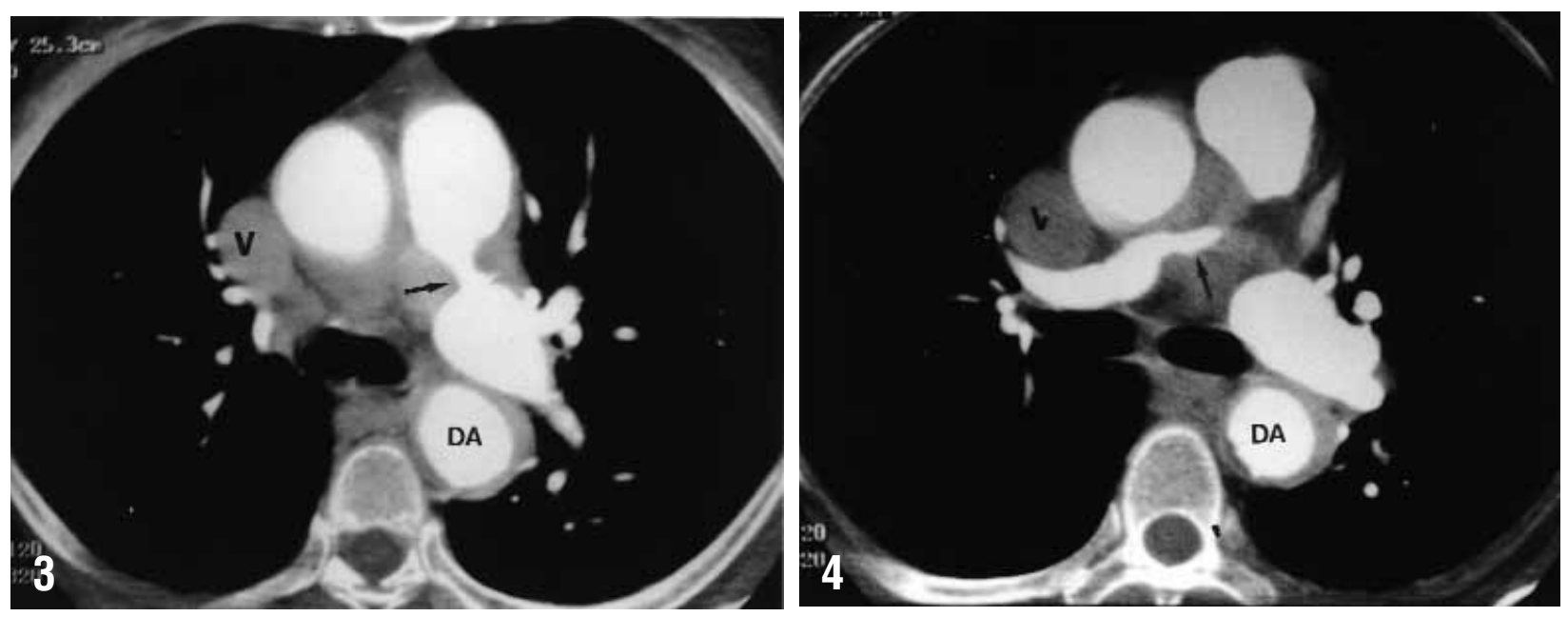

Figs 3 and 4. The superior vena cava $(V)$ is dilated. Focal narrowing and thickening of the walls of the left pulmonary artery and right pulmonary artery (arrows) is seen. Thickening of the wall of the descending aorta $(D A)$ is also noted. No pulmonary emboli are seen.

ferential diagnosis would also include fibrosing mediastinitis, but in most of those cases there would be more evidence of a soft tissue mass or infiltration of the mediastinum with lymph node calcifications.

Dr Patterson: Dr Glazer, could you make a comment about CT scans in the evaluation of pulmonary embolism? What is the role of the pulmonary angiogram? What is the role of CT imaging?

Dr Glazer: Helical CT has increasingly been used to evaluate patients in whom pulmonary emboli are suspected. It can accurately diagnose emboli in segmental or larger vessels. Although it is not as accurate in identifying subsegmental emboli, improvements in technique should allow more accurate identification of these emboli. Moreover, the clinical importance of isolated subsegmental emboli is uncertain. CT has been particularly helpful in those cases in which the ventilation-perfusion scan indicates an intermediate probability of pulmonary embolism or in which multiple abnormalities are seen on the chest radiograph. Most important, the role of helical CT should be based on the results of multicenter outcome studies that compare its value relative to more conventional approaches. Dr Siegel, would you like to comment?

Dr Siegel: Overall, your answer is quite balanced, given the state of flux of our knowledge. There need to be better controlled multicenter comparison studies of ventilation-perfusion imaging and CT. The National Heart, Lung, and Blood Institute has recently decided to fund a study labeled "PIOPED II," in which we will be participating. This study as proposed is primarily a comparison of helical CT with ventilation-perfusion imaging, with a substantial focus on outcome data rather than just the correlation with the results of pulmonary arteriography. In the final analysis, outcome data are much more important. It has been known for quite a long time that some fraction of patients with socalled "low-probability" lung scans actually have small pulmonary emboli, typically in the subsegmental vessels. Depending on one's perspective, these could be considered "false-negative" lung scans. On the other hand, a moderately large body of data shows if untreated patients with low-probability lung scans are monitored, the frequency of subsequent events attributable to pulmonary embolism is at a very low level. ${ }^{1}$ The concept of clinically insignificant pulmonary embolism is thus suggested. It has been argued that the pulmonary circulation was designed the way it was, with rich collateral connections at the microvascular level, in part to remove the "detritus" coming from the lower extremities. Despite this, ventilation-perfusion studies have been criticized because of these apparent "misses." Now CT comes on the scene and the data show that CT cannot evaluate those small vessels. The mantra is that those small emboli are not important. I think we are going to find as we gather more data that the truth lies somewhere in the middle. There is likely to be a subset of patients we can evaluate very quickly and very safely, with a lower radiation dose, lower expense, and no need for iodinated contrast agents by use of ventilation-perfusion imaging, whereas in other patients it will be entirely appropriate to proceed directly to CT.

Dr Glazer: It is important that the clinicians discuss their individual cases with the radiologist to determine which imaging test is most appropriate depending on the clinical circumstances. 
Dr Cooper: That is especially true in patients with emphysema who often have a number of ventilation defects on nuclear lung scan. My sense is if a CT scan is positive it might be more convincing in this particular setting than an indeterminate ventilation-perfusion scan. For patients who do not have postoperative x-ray changes showing infiltrates or effusions, I do not know which is better.

Dr Safdar: Which patients do you recommend have ventilation-perfusion scans and which patients do you recommend undergo spiral CT scan?

Dr Siegel: I do not know the exact answer to that. Certainly the average patient with symptoms suggesting acute pulmonary embolism who has a relatively normal chest radiograph and no known history of severe chronic lung disease is the perfect candidate for obtaining a quick answer with ventilation-perfusion imaging. The decision becomes more complicated in the presence of radiographic infiltrates or large pleural effusions. In those cases, it may be reasonable to proceed directly to spiral CT. However, remember how this clinical scenario plays out now in many cases. Results of the lung scan are abnormal because of the radiographic abnormalities. By the strict criteria, we interpret the study as indicating an intermediate probability of pulmonary embolism. The referring physician then reasons that the patient was really believed to have pneumonia or congestive heart failure, but the lung scan was obtained to exclude pulmonary embolism as an alternative treatable cause of the signs and symptoms. The vast majority of such patients do not undergo pulmonary arteriograms, and we do just fine in treating them. We are not missing large numbers of patients with hidden pulmonary embolism by use of this strategy. Although it is obviously reasonable to try to get to a more precise diagnosis in as many patients as possible, we should not fault ourselves if we use good clinical judgment and have a good outcome despite some level of diagnostic uncertainty.

Dr Safdar: I agree. If there is an intermediate probability ventilation-perfusion scan, would you do a spiral CT scan or would you go to a pulmonary angiogram?

Dr Siegel: I would probably do a spiral CT under those circumstances. In fact, much of the literature argues that spiral CT is more likely to supplant pulmonary arteriography for definitive evaluation than it is to supplant ventilation-perfusion scintigraphy as the screening test. A study published by Wells and associates $^{2}$ from McMaster University in 1998 shows that by use of relatively simple pieces of readily available clinical data for assessment of the prior probability of pulmonary embolism, in combination with ventilation-perfusion imaging, a strategy for diagnostic evaluation and management can be devised in which arteriography will be needed in only a small percent of patients and in which outcome is very good. This exemplifies the type of strategy that we need to use; we need to apply it to spiral CT, we need to apply it to ventilation-perfusion imaging, and we need to look at various costs (including radiation doses). I believe that when this type of analysis has been done, we will end up with some level of use of both tests. Right now we do not have good enough data to tell you precisely what you ought to be doing, and many current opinions are dogmatic and not based on hard evidence.

Dr Safdar: In this institution, if the house staff suspects pulmonary embolism they request a spiral CT. Do you have any idea what percent of those turn out to be positive?

Dr Glazer: Approximately 20\%.

Dr Siegel: We are still doing between 10 and 15 ventilation-perfusion studies daily.

Dr Glazer: Approximately 3 to 5 helical CT pulmonary embolism protocols are performed per day.

Dr Sundt: I should also note that Dr Glazer and his colleagues have been quite interested in exploring the use of spiral CT scanning with 3-dimensional reconstruction in the evaluation of our patients with chronic thromboembolic disease before undertaking thromboendarterectomy.

Dr Siegel: I have just one more point about the case at hand. I have not seen the original interpretation of the outside lung scan, but if the CT was done to evaluate for pulmonary embolism based on the results of the lung scan, this was not really appropriate. The lung scan, as Dr Yu indicated, should have been interpreted as most consistent with a disorder causing extrinsic obstruction of either the pulmonary arteries or pulmonary veins rather than pulmonary embolism. Unilateral whole-lung hypoperfusion is a very atypical pattern for pulmonary embolism.

Dr Glazer: In some cases, the ventilation-perfusion scan is the initial test to suggest a central obstructing mass by demonstrating unilateral hypoperfusion.

Dr Pechet: The next step in the patient's workup was a right heart catheterization, which demonstrated pressures of $80 / 25 \mathrm{~mm} \mathrm{Hg}$ in the proximal pulmonary artery. Bilateral pulmonary artery stenoses, $80 \%$ left and $90 \%$ right, were identified with a $50 \mathrm{~mm} \mathrm{Hg}$ gradient. The patient was then taken to the operating room with a presumed diagnosis of fibrosing medias- 


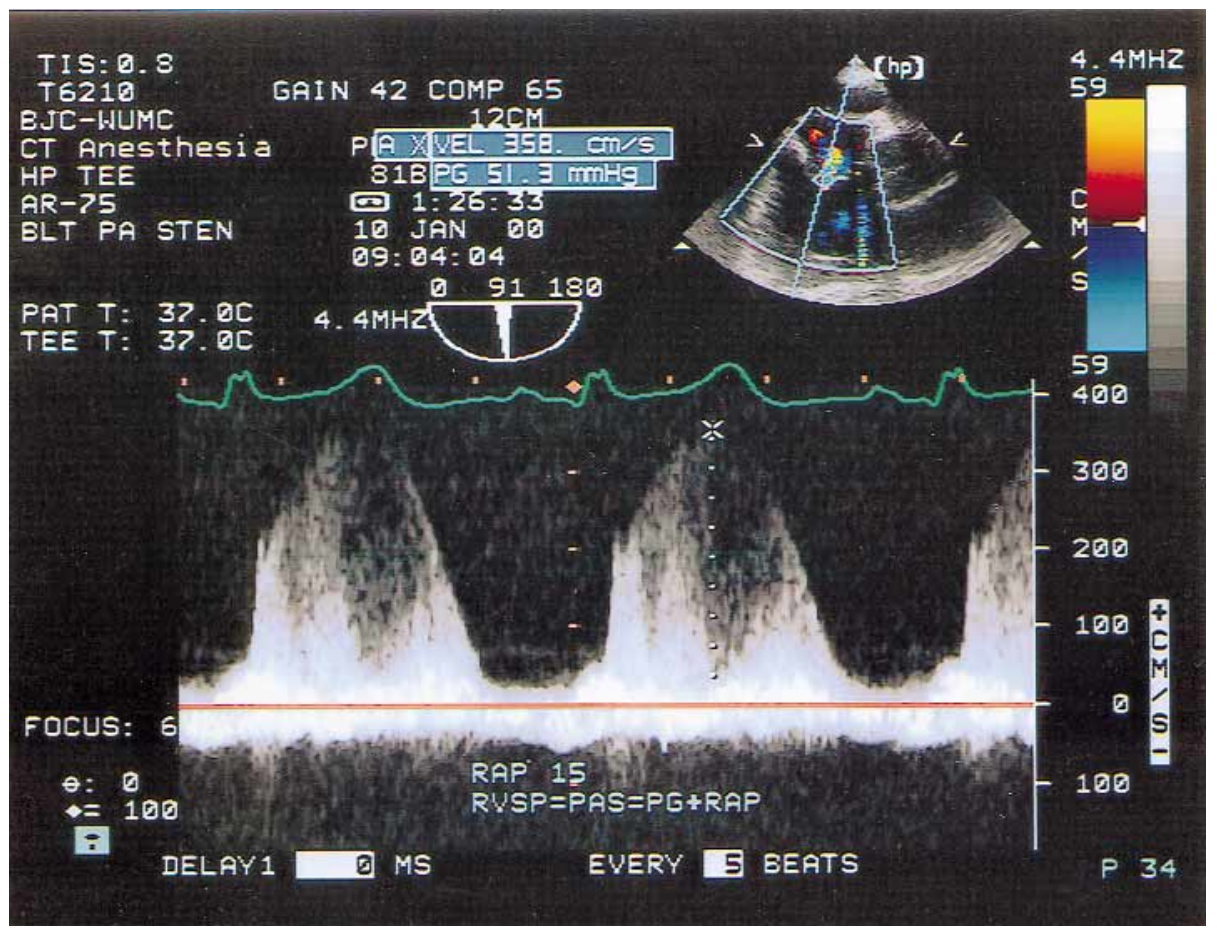

Fig 5. Continuous wave Doppler TEE before repair depicting tricuspid regurgitation. The estimated right ventricular end-systolic pulmonary artery pressure is $66 \mathrm{~mm} \mathrm{Hg}$.

tinitis for a cervical mediastinoscopy. The pathology report revealed only peripheral nerve. The diagnosis at this point was that of neurogenic tumor. The patient was subsequently referred to Dr Cooper.

Dr Cooper: The outside pathologist saw neural tissue and was considering a diagnosis of neurosarcoma. The outside slides were reviewed here.

Dr Ritter: Eight negative lymph nodes specimens were submitted. In addition, a specimen labeled "left paratracheal mass" showed some benign lymph node tissue and an unremarkable peripheral nerve.

Dr Cooper: I asked the surgeon whether by any chance the patient was hoarse after the mediastinoscopy, and he said, as a matter of fact, yes.

Dr Pechet: At that time she was transferred here. She underwent her first transthoracic echocardiogram, which demonstrated no abnormalities of the left side of the heart. The right side of the heart was markedly dilated and hypokinetic. She had moderate tricuspid regurgitation and a $60 \mathrm{~mm} \mathrm{Hg}$ gradient immediately above the pulmonary valve. After this she was taken to the operating room by Dr Cooper and Dr Sundt for mediastinal exploration.

Dr Cooper: After the sternotomy, no gross abnormalities were visible. On palpating the main pul- monary artery from its origin to the bifurcation, we encountered a point at which the artery became very firm with a rigid, thick shelf within the wall of the artery. This felt like a mass within the wall of the pulmonary artery itself. Most important, when palpating outside the pulmonary artery, around it and around the left and the right pulmonary arteries, we detected no process extending outward from the adventitia. That is, there was a normal plane between the artery and the surrounding tissue. The first few centimeters of the pulmonary artery seemed normal on palpation, and after a few centimeters this thick shelf could be felt inside the lumen. However, there was no inflammation or fibrosis outside the artery. I have never seen fibrosing mediastinitis involve only the lumen of the vessel and not extend into the vessel from a surrounding inflammatory mass, which was not present here.

Dr Patterson: Was a transesophageal echocardiogram (TEE) performed in the operating room?

Dr Skubas: Invasive intraoperative monitoring included an arterial line, a central venous pressure line, and TEE. TEE before repair revealed dilatation of the hepatic veins, right atrium (transverse diameter $7 \mathrm{~cm}$ ), and coronary sinus and an interatrial septum that was bulging toward the left. There was $1+$ central jet of tri- 


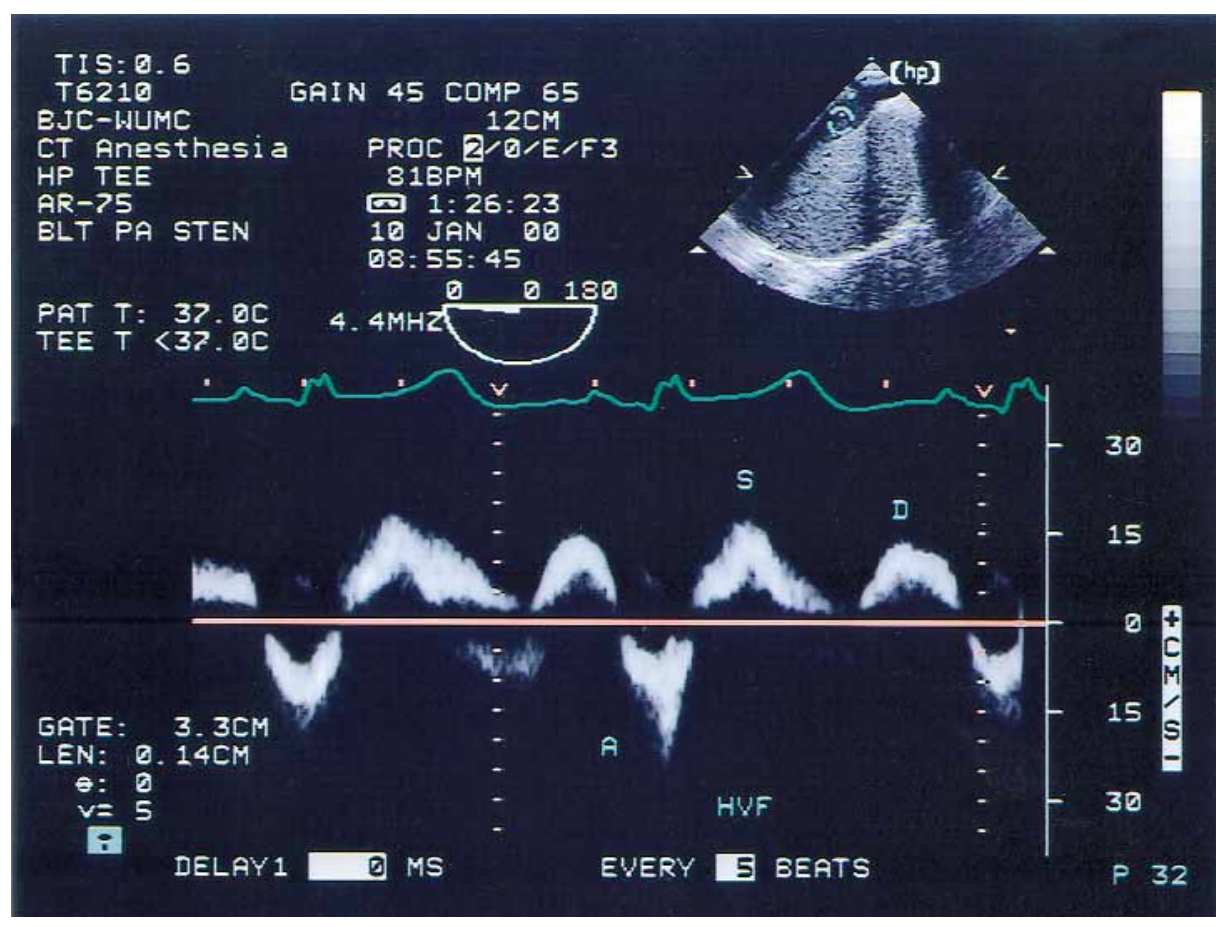

Fig 6. Continuous wave Doppler TEE before repair of the hepatic veins. The systolic $(S)$ to diastolic $(D)$ ratio is greater than 1.

cuspid regurgitation, and the systolic pulmonary artery pressure was calculated to be $66 \mathrm{~mm} \mathrm{Hg}$ (Fig 5). Examination of the main pulmonary artery and its bifurcation did not reveal any thrombus. Doppler examination revealed impaired early diastolic filling of the right ventricle (hepatic venous flow systolic/diastolic ratio greater than 1; Fig 6). The left ventricular filling pattern revealed a transmitral early to late velocities ratio less than 1 and a pulmonary venous flow systolic/diastolic velocities ratio greater than 1 , consistent with relaxation abnormality.

Dr Sundt: We performed the operation using normothermic cardiopulmonary bypass without cardioplegic arrest. We thought that this approach would provide us with optimal exposure for diagnostic purposes and would allow us to be certain that we were constructing our anastomoses in regions free of disease. We were thereby also able to avoid placing clamps on these fragile vessels. Previously, we have relieved pulmonary artery obstruction due to sarcoma by endarterectomy with good results; however, in this case we were reluctant to attempt the same in the absence of a diagnosis. We were also concerned that there might not be a satisfactory dissection plane and that we would create an unreconstrucible mess. It seemed more straightforward to simply place separate ringed polytet- rafluoroethylene (PTFE) grafts* to each distal pulmonary artery from the proximal main pulmonary artery.

The distal anastomosis to the left pulmonary artery was at the pericardial reflection approximately $1 \mathrm{~cm}$ beyond the gross disease. We placed the distal anastomosis to the right pulmonary artery between the SVC and the aorta. This also was well beyond the gross disease, and we thought that placing it medial to the SVC offered several advantages: it put us proximal to the takeoff of the right upper lobe branch and therefore still in a segment of the vessel with a large diameter, and it avoided the risk of compression of the SVC between the ringed PTFE graft and the aorta.

The proximal anastomosis of each graft was to the main pulmonary artery. When we opened the proximal main pulmonary artery, the wall itself appeared entirely normal, but just distal to the arteriotomy we could see a bluish mass within the lumen involving the back wall and the more distal main pulmonary artery. We took multiple, deep biopsy specimens of the mass and sent them for frozen section examination. Because the

*Gore-Tex graft; registered trade name of W. L. Gore \& Associates, Inc, Flagstaff, Ariz. 


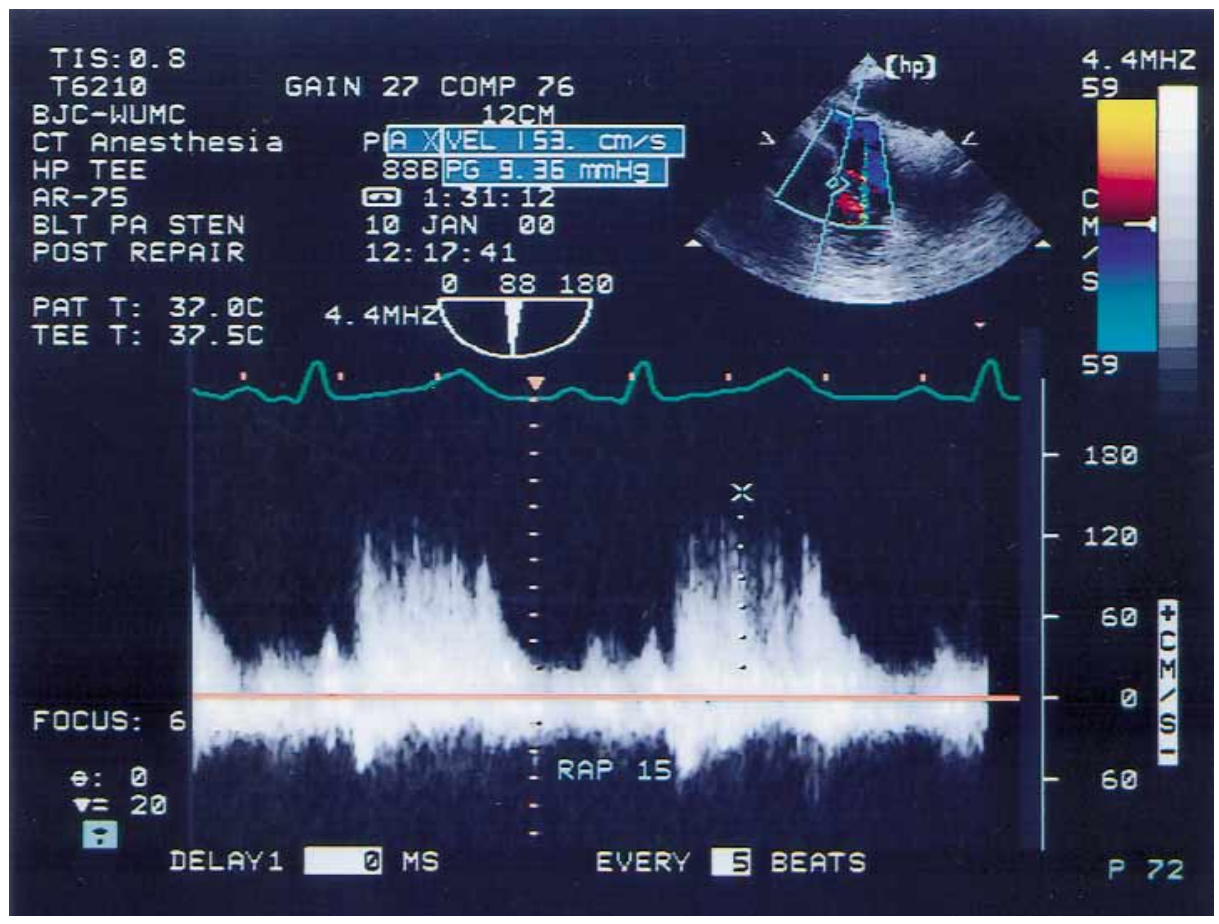

Fig 7. Continuous wave Doppler TEE after repair. The tricuspid regurgitation jet is significantly reduced. The estimated systolic pulmonary artery pressure is $24 \mathrm{~mm} \mathrm{Hg}$.

proximal main pulmonary artery did not appear involved, we used our arteriotomy site for one of the proximal anastomoses and created a second arteriotomy for the other graft. Although the potential for extension of the disease process to involve these sites exists, we thought that this was simpler than proximal anastomosis to the right ventricle itself and that it preserved a valve in the system.

Dr Skubas: TEE examination after the repair revealed a decrease in the size of the right atrium, with minimal (trace) tricuspid regurgitation, and the calculated systolic pulmonary artery pressure was decreased to $24 \mathrm{~mm} \mathrm{Hg}$ (Fig 7). Doppler examination demonstrated a marked recovery of the early diastolic filling of both the right ventricle (hepatic venous flow systolic/diastolic velocities ratio less than 1, Fig 8) and left ventricle (transmitral early to late ratio greater than 1 and pulmonary venous flow systolic/diastolic ratio less than 1).

The TEE was used as a quantitative and qualitative diagnostic tool in this case. First, because of the bilateral pulmonary artery stenoses, a pulmonary artery catheter was not inserted, and the TEE Doppler examination was used to calculate the systolic pulmonary artery pressure before the procedure, as well as after the repair. In this way, TEE proved the adequacy of repair, as it demonstrated decrease in pulmonary artery pressures after the bypass. Second, the TEE examination demonstrated the relative clinical signs of increased afterload of the right ventricle: dilatation of the right atrium, hepatic veins, and coronary sinus, as well as tricuspid regurgitation. Third, the TEE examination of the hepatic venous flow and the pulmonary venous and transmitral flow revealed improved filling of the right and left ventricles, respectively.

Dr Pechet: Fig 9 shows an operative photograph depicting what Dr Sundt described. The right and left end-to-side 12-mm ringed PTFE bypass grafts to the distal pulmonary artery are seen. Postoperatively, the patient did quite well and was discharged home on postoperative day 4 after medialization of the vocal chord. Dr Ritter reviewed the pathology for us.

Dr Ritter: The frozen specimen was really quite interesting, with the expectation that perhaps this was going to be a pulmonary artery sarcoma. When we did the frozen section, it showed the media of the artery wall (Fig 10), which accounts for the fact that the specimen looks "striped," with inflammatory cells just tracking through along the pre-existing elastic tissue of the media. That is all that the frozen section showed; 


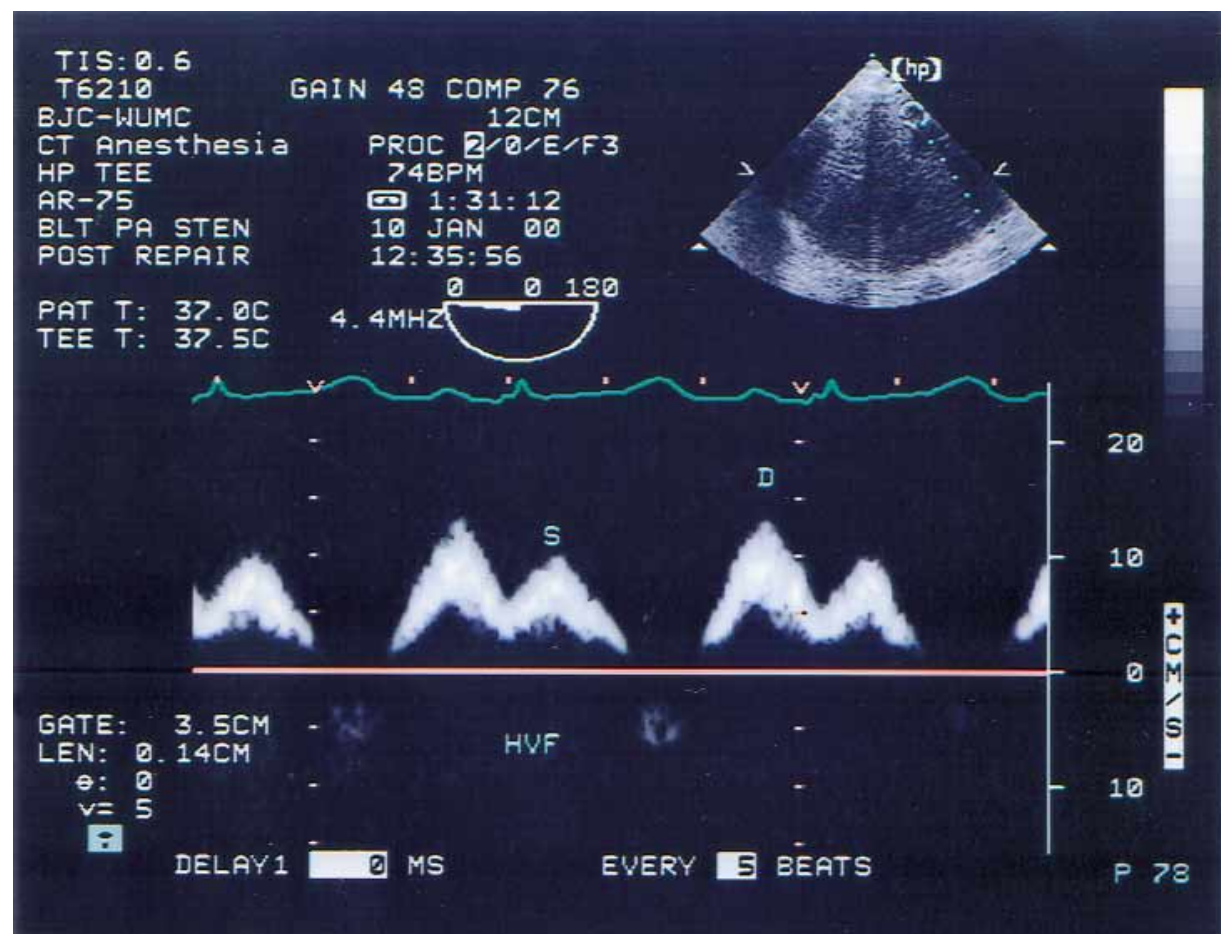

Fig 8. Continuous wave Doppler TEE after repair of the hepatic venous flow $(H V F)$. The systolic/diastolic $(S / D)$ ratio is now less than 1.0.

there was nothing that looked neoplastic. Fig 11 shows more of this inflammatory tissue, which is fibrotic, suggesting that it had been present for some time. The specimen actually contained small vessels, which are in tissue that Dr Cooper assured us came from the inside of the pulmonary artery. Thus, some small systemic arteries have actually formed in the pulmonary artery lumen. In other areas the tissue showed the sort of foam-cell change that might be seen in an atherosclerotic plaque (Fig 12). On the permanent sections of the tissue that had been frozen are plasma cells; Fig 13 shows their eccentric nucleus and amphophilic cytoplasm. At the time that we saw the frozen section, I did not think this was neoplastic; we were considering perhaps an inflammatory pseudotumor, which has rarely been reported to occur in the lung as an intra-arterial lesion and can actually mimic pulmonary emboli. Nonetheless, we were not completely sure what we were dealing with when we were examining the frozen section.

When we got to the permanent sections, the picture became more clear. There were abscess-like areas, with some granulomas and some palisading histiocytes (Fig 14). Again, we observed giant cells and areas of necrosis. Fig 15 shows an area of necrosis with some puru- lent debris containing neutrophils and then a palisade of histiocytes and giant cells around the outside of this focus. This was located right in the pulmonary artery media, so it is actually an area of medial necrosis with a granuloma around it. Some of these necrotic areas appeared to contain pieces of elastic tissue; that occurs with necrosis of a vessel wall. Therefore, we thought this was a granulomatous arteritis. The types of large vessel arteritis that can develop in the pulmonary arteries are all quite unusual but include Takayasu arteritis, giant cell arteritis, rheumatoid vasculitis, and even Wegener granulomatosis. With the granulomas, acute inflammatory foci, and fibrosis, I think this looks histologically most like Takayasu arteritis. It certainly is an unusual presentation, since almost all patients with Takayasu arteritis have been reported to be younger than 40 or certainly younger than 50 years of age. Nonetheless, with apparent involvement of the aorta and the histologic characteristics, the evidence looks reasonably convincing for that entity.

Dr Cooper: Dr Glazer, there were two CT scans, 6 months apart. Did you review those?

Dr Glazer: No.

Dr Cooper: We had gotten the old CT scan from August and could see no changes between the two, 
Volume 121, Number 1

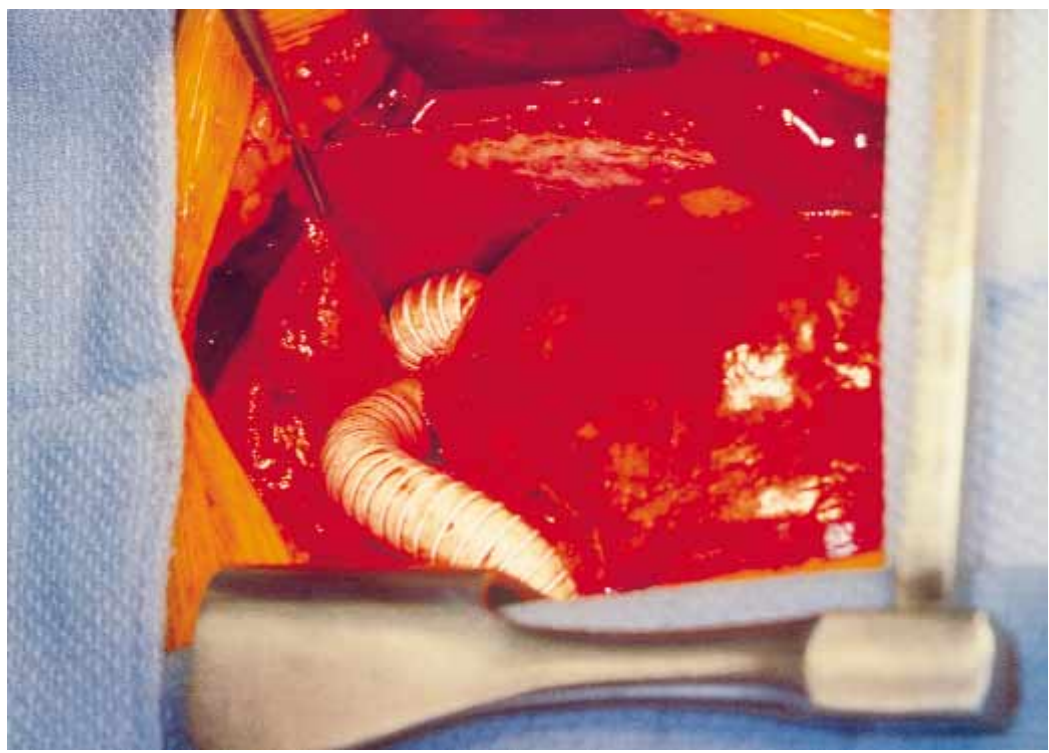

Fig 9. Operative photograph shows the separate ringed PTFE grafts from the main pulmonary artery to the right and left pulmonary arteries (head is to the left).

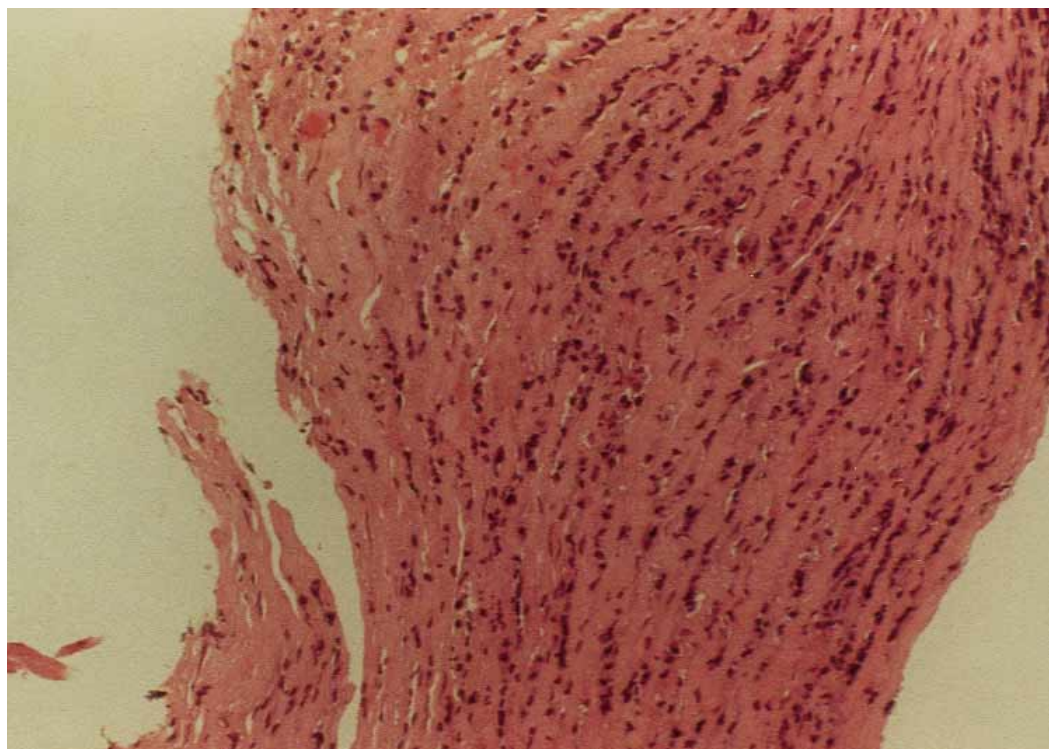

Fig 10. Tissue submitted for frozen sections showed inflammatory cells in arterial media; the tracking of inflammatory cells between the elastic and muscular layers imparts a "striped" appearance.

which encouraged us. I asked her family how long in retrospect they thought that she had been symptomatic. They said she used to tool around her large farm with no problem at all; in the past 3 or 4 years there had been a decline, but for a woman in her mid-70s, it is hard to know how much can be attributed to advancing years. However, she had changed from a very energetic active person to someone who could hardly walk.
Dr Roper: I have a question for pathology. I have seen only one primary tumor in the pulmonary circulation, and that was a number of years ago in a patient from Columbia, Missouri. His left pulmonary artery tumor was termed a hemangiopericytoma. What would the microscopic examination reveal? I have seen mediastinal fibrosis involving the pulmonary artery. 


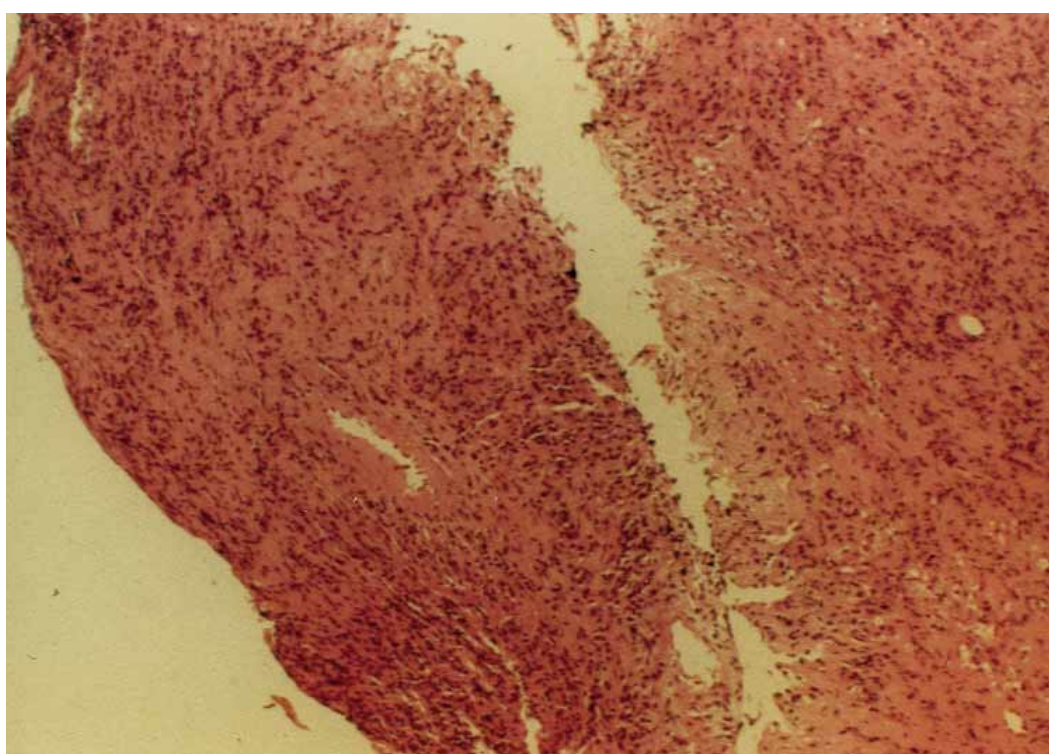

Fig 11. Additional frozen section tissue showed inflamed fibrous tissue, as well as the presence of systemic capillaries.

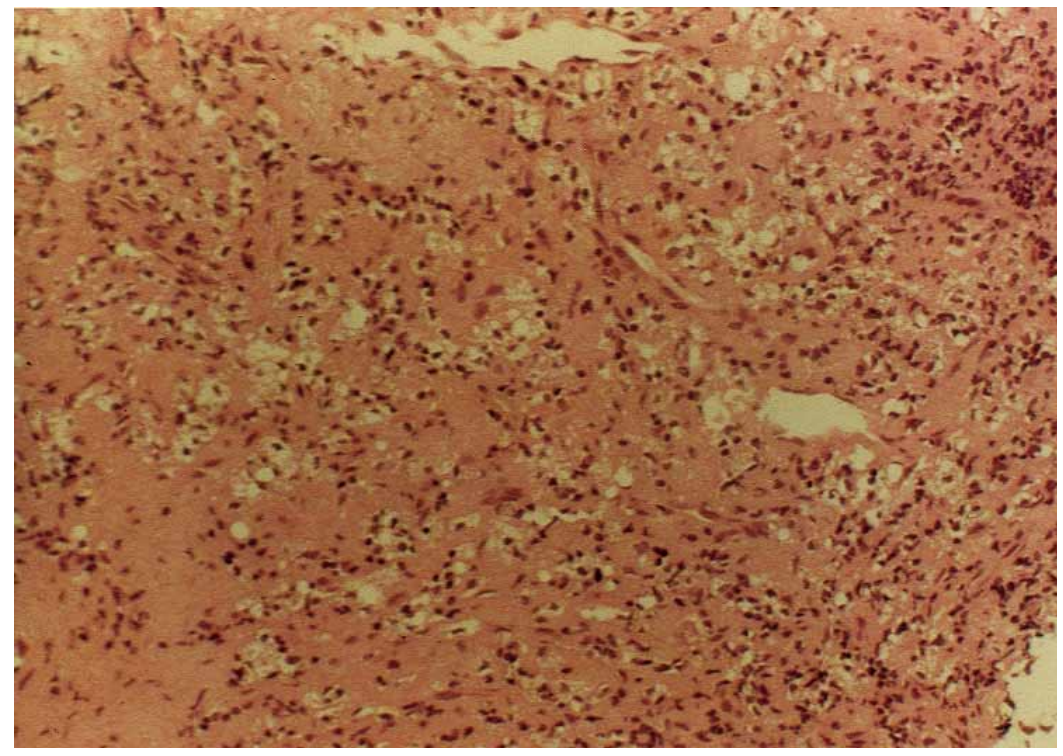

Fig 12. Sections of the intra-arterial tissue showed inflamed fibrous tissue, as well as foamy histiocytes.

Dr Ritter: The pulmonary artery sarcomas are really a mixed bag of tumors. Most of them look like some type of smooth muscle tumor-leiomyosarcoma, for example. Other tumors can only be called high-grade spindle cell malignant tumors. Others may resemble hemangiopericytoma.

Dr Roper: That was the diagnosis in the one I saw.

Dr Ritter: Another concern about these so-called pulmonary artery sarcomas is whether they might be metas- tases that get lodged in the pulmonary artery. Once that possibility has been excluded, a variety of conditions get lumped under the term pulmonary artery sarcoma that represent a variety of soft tissue sarcomas.

Dr Cooper: Dr Glazer, how did you interpret the thickening around the descending aorta? We noticed in advance a concentric thickening of the aortic wall.

Dr Glazer: Thickening of the wall of the descending aorta, in association with thickening of the walls 


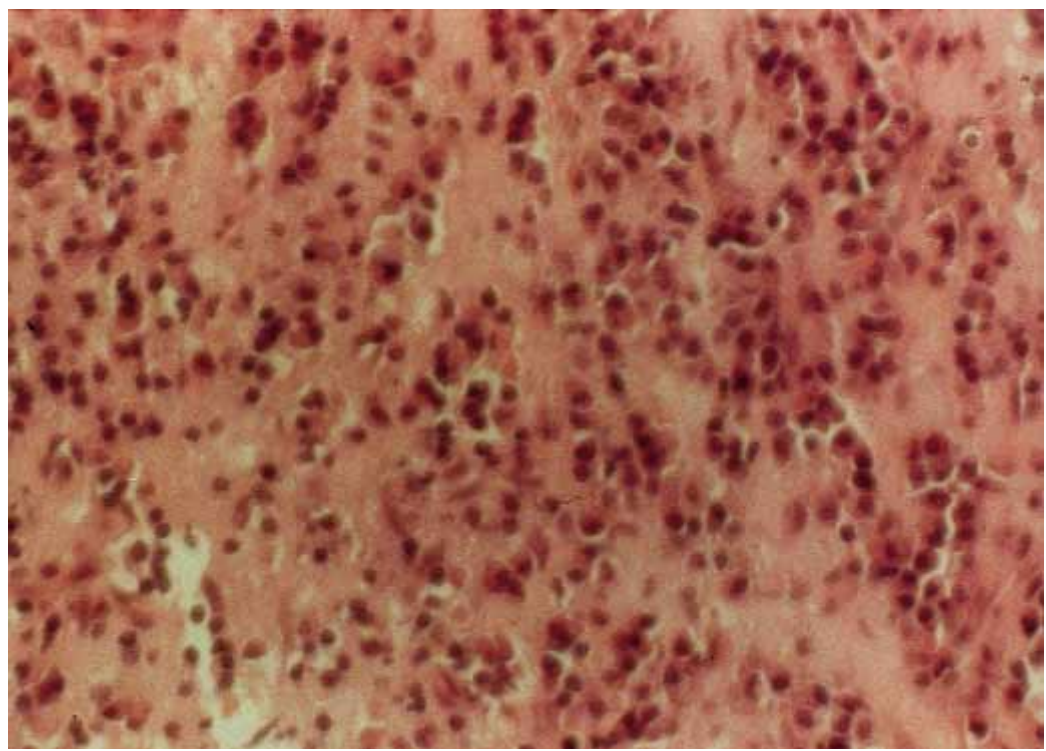

Fig 13. Abundant plasma cells were present within the media; typical features of plasma cells include an eccentric nucleus, amphophilic cytoplasm, and perinuclear halos.

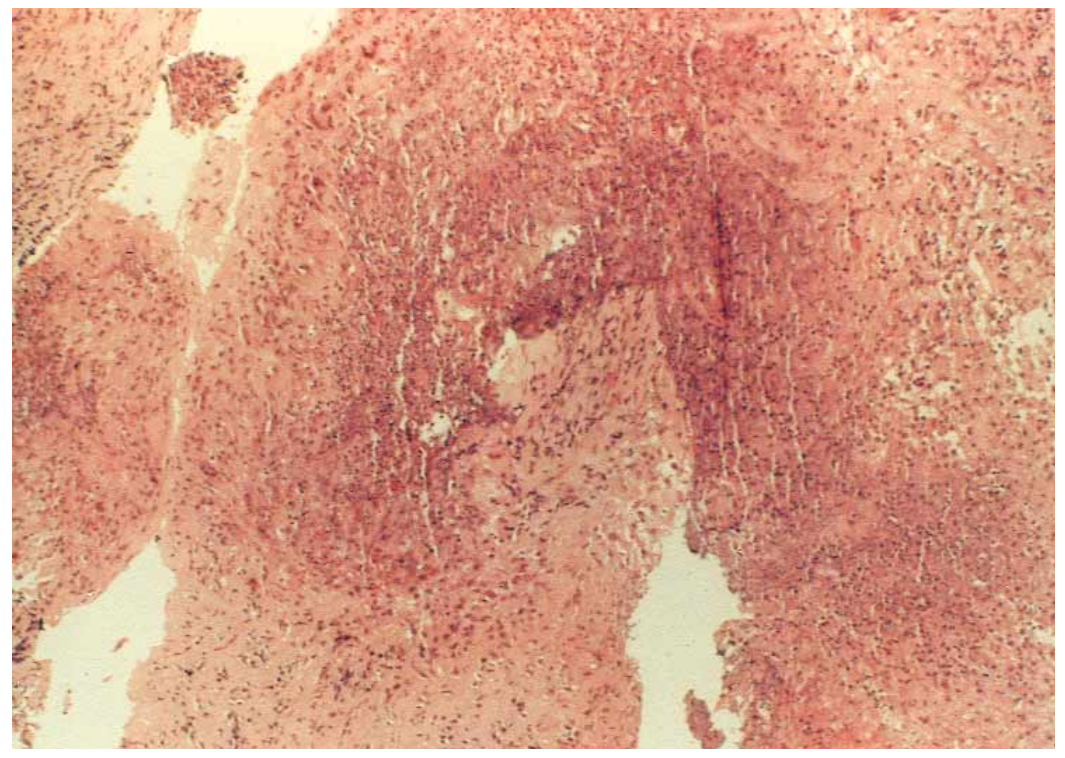

Fig 14. Necrosis with abscess-like pattern, including neutrophils in the necrotic debris. There is a surrounding palisade of histiocytes.

of pulmonary arteries, is highly suggestive of a vasculitis.

Dr Cooper: Is that an acceptable radiologic picture for Takayasu arteritis?

Dr Glazer: Yes.

Dr Cooper: I was initially worried that we missed something, that maybe the biopsies were not deep enough.
Dr Ritter: No, you have clearly gotten into the media, because those inflammatory cells can be seen tracking through the media and there are necrotizing granulomas in the middle of that. I am pretty sure this is representative of the pathologic process.

Dr Patterson: How is Takayasu arteritis treated?

Dr Pechet: The primary treatment for Takayasu arteritis is immunosuppression. The response rate to 


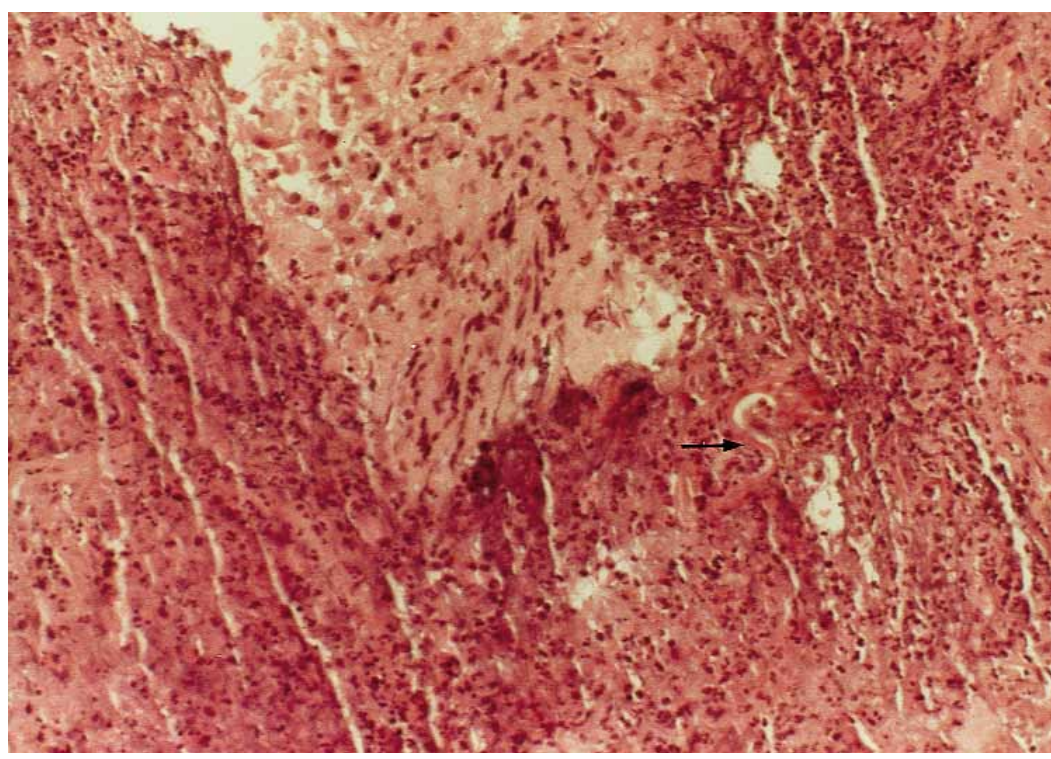

Fig 15. A higher power view shows necrotic and purulent debris, with surrounding histiocytes and giant cells. There is a serpentine elastic fiber with the necrotic debris (arrow).

glucocorticoids is highly variable, ranging from $20 \%$ to $100 \%$. In patients with steroid-resistant disease, methotrexate, cyclophosphamide, and azathioprine have all been used with some success.

Dr Patterson: Is it a lethal condition?

Dr Pechet: Takayasu arteritis is rarely fatal, although myocardial failure, renal failure, stroke, and aneurysm rupture have been reported as causes of death.

Dr Sundt: Occasionally patients will die of the sequela of their occlusive disease. Most often, the brachiocephalic vessels are involved and patients may undergo synthetic graft reconstruction for cerebrovascular insufficiency. Unfortunately, the condition may be progressive, with ultimate occlusion of those grafts with anastomotic or distal disease. It may also lead to lower extremity or visceral ischemia necessitating peripheral vascular bypass.

Dr Cooper: What fraction of patients have involvement primarily of the pulmonary arteries?

Dr Ritter: As Dr Glazer said, involvement of the pulmonary arteries has been reported and the number or percentage of patients who have pulmonary artery involvement varies from study to study. In some of the Japanese studies, in which pulmonary angiograms have been obtained on everybody with the diagnosis, most of the time the patients had some kind of involvement of the pulmonary arteries, although clinically that would not necessarily have been suspected.

Dr Pechet: As Dr Ritter said, most of this literature is from Japan. Although a wide range has been report- ed, approximately $50 \%$ of cases of Takayasu arteritis involve the pulmonary artery. ${ }^{3}$ There are also cases of isolated pulmonary artery involvement at presentation. ${ }^{4}$ Most often, the disease is more peripheral than in this case.

Dr Roper: Dr Siegel, what do ventilation-perfusion scans typically show in Takayasu arteritis? Is this case typical?

Dr Siegel: I have not seen a presentation like this. We have seen several cases of Takayasu arteritis, and they have involved lobar and segmental pulmonary arteries; the appearance simulates that of pulmonary embolism, as reported by others. ${ }^{5}$

Dr Cooper: How were these cases diagnosed, by biopsy or excision?

Dr Siegel: They were diagnosed by arteriography after ventilation-perfusion imaging. I do not know how many of those cases involved biopsies.

Dr Cooper: Then how could you make the diagnosis?

Dr Siegel: The arteriogram shows a typical smooth region of narrowing involving a long segment of the artery. This appearance suggests arteritis, and largevessel involvement is typical of Takayasu arteritis. The patients we have seen were the typical young women with no evidence of other problems to suggest another systemic vasculitis, such as polyarteritis nodosa, which can have similar pulmonary arterial involvement. In the cases I recall, there also was aortic or aortic branch involvement and the ventilation-perfusion findings looked like those of pulmonary embolism. 
Dr Patterson: This case was presented yesterday at the chest radiology conference. The point was made that in patients who were known to have Takayasu arteritis, a detailed prospective search of the pulmonary circulation shows that the incidence of pulmonary involvement is really very high. Does this patient have aortic involvement too?

Dr Cooper: Yes. We knew that the aortic wall was abnormal, but we did not know what the abnormality was and whether it was related to the obstructing pulmonary artery lesions. I had been sent the CT scans in advance, and I thought the appearance suggested a sarcoma. When the patient decided to come here, I told her doctors that (1) I could not promise that we could do any more at all and she might waste a trip and (2) if we did do something surgical for her she might add only 1 or $1 \frac{1}{2}$ years to her life because the tumor was probably a slowly growing sarcoma and the best we could do was to buy some time for her.

Dr Sundt: She does not, however, have known involvement of the brachiocephalic vessels.

Dr Roper: Will you prescribe anticoagulation for the rest of her life?
Dr Pechet: There is not an established role for anticoagulant or antiplatelet therapy in treating Takayasu arteritis. She has, however, been placed on a regimen of long-term warfarin (Coumadin) therapy to maintain patency of the bypass graft.

\section{REFERENCES}

1. Lee ME, Biello DR, Kumar B, Siegel BA. "Low-probability" ventilation-perfusion scintigrams: clinical outcomes in 99 patients. Radiology 1985;156:497-500.

2. Wells PS, Ginsberg JS, Anderson DR, Kearon C, Gent M, Turpie AG, et al. Use of a clinical model for safe management of patients with suspected pulmonary embolism. Ann Intern Med 1998;129:997-1005

3. Haque U, Hellmann D, Traill T, Venbrux A, Stone J. Takayasu's arteritis involving proximal pulmonary arteries and mimicking thromboembolic disease. J Rheumatol 1999;26:450-3.

4. Lie JT. Pathology of isolated nonclassical and catastrophic manifestations of Takayasu arteritis. Int J Cardiol 1998;66(Suppl 1):S11-21

5. Umehara I, Shibuya H, Nakagawa T, Numano F. Comprehensive analysis of perfusion scintigraphy in Takayasu's arteritis. Clin Nucl Med 1991;16:352-7. 